\title{
Haptic Edge Sharpness Perception with a Contact Location Display
}

\author{
Jaeyoung Park, Student Member, IEEE, Andrew J. Doxon, Student Member, IEEE, \\ William R. Provancher, Member, IEEE, David E. Johnson, and \\ Hong Z. Tan, Senior Member, IEEE
}

\begin{abstract}
The effect of contact location information on virtual edge perception was investigated in two experiments. In Experiment 1 , participants discriminated edge sharpness under force-alone and force-plus-contact-location conditions using a $4.8 \mathrm{~mm}$ radius contact roller. Virtual objects were 2D profiles of edges with two adjoining surfaces. For both conditions, the Just Noticeable Difference (JND) in change of edge radius increased from 2.3 to $7.4 \mathrm{~mm}$ as edge radii increased from 2.5 to $20.0 \mathrm{~mm}$; there was no significant difference between the two conditions. A follow-up experiment with contact location alone resulted in higher edge sharpness JNDs. In Experiment 2, the same edge sharpness discrimination task was performed using a smaller contact roller $(R=1.5 \mathrm{~mm})$ to investigate the effect of roller size. The JNDs for the smaller roller were not statistically significant from those of the larger roller. Our results suggest that 1) contact location cues alone are capable of conveying edge sharpness information, but that force cues are dominant when both types of cues are available; and 2) the radius of the contact roller does not significantly affect the user's ability to discriminate edge sharpness, indicating that the participants could use the changes in contact location to judge curvature.
\end{abstract}

Index Terms-Contact location display, edge sharpness perception, curvature discrimination, effect of contact element size

\section{INTRODUCTION}

$\mathrm{T}$ HE present study is part of an ongoing research program to develop a contact location display (CLD) that can adequately complement a force-feedback device. This tactile display provides cutaneous contact information during manual interaction between a single finger and a virtual environment. While force-feedback displays have been successful at conveying kinesthetic force information from a single contact point, they lack the distributed stress and strain information that is critical for precision grip and interaction with small-scale surface features. A contact location display has been developed as a first step toward a haptic system capable of displaying both cutaneous and kinesthetic information to the fingertip [1].

The present study investigates the extent to which the CLD benefits the exploration and perception of shape primitives in touch. As shown by van der Horst and Kappers, curvature information plays an important role in

- J. Park is with the Haptic Interface Research Laboratory, Purdue University, Electrical Engineering Building, 465 Northwestern Ave., Mailbox 303, West Lafayette, IN 47907-2035. E-mail: park183@purdue.edu.

- A.J. Doxon and W.R. Provancher are with the Department of Mechanical Engineering, University of Utah, 50 S. Central Campus Dr., Merril Engineering Building, Salt Lake City, UT 84112. E-mail: adoxon@gmail.com,wil@mech.utah.edu.

- D.E. Johnson is with the School of Computing, University of Utah, 72 Central Campus Dr., 2875 Warnock Engineering Building, Salt Lake City, UT 84112. E-mail: dejohnso@cs.utah.edu.

- H.Z. Tan is with the Haptic Interface Research Laboratory, Purdue University, Electrical Engineering Building, 465 Northwestern Avenue, West Lafayette, IN 47907-2035. E-mail: hongtan@purdue.edu.

Manuscript received 15 July 2011; revised 24 Feb. 2012; accepted 28 Feb. 2012; published online 7 Mar. 2012.

Recommended for acceptance by A. Kappers.

For information on obtaining reprints of this article, please send e-mail to: toh@computer.org, and reference IEEECS Log Number TH-2011-07-0051.

Digital Object Identifier no. 10.1109/ToH.2012.14. haptic shape perception [2]. Corners of varying degrees of sharpness can be regarded as important local geometrical surface features for the haptic perception of object. We therefore selected corner sharpness perception as the focus of the present study. Because the current prototype of the CLD is 1-DOF, it is technically more accurate to describe the objects used in the present study as two flat surfaces connected with an extruded 2D circular arc representing an edge of controllable sharpness. Our specific objectives were to 1) measure the relative contributions of force and contact location information in edge sharpness perception, and 2) to assess the effect of contact roller size on sharpness perception of virtual edges. For the first objective, we hypothesized that the availability of contact location information, enabled by the actuated roller on the CLD that simulated the movement of a virtual edge on the fingertip, should enhance a user's ability to discriminate the sharpness of virtual edges. For the second objective, we hypothesized that a smaller contact roller may lead to a better discrimination performance, due to a greater localization of the moving contact represented by the smaller roller. Two experiments were conducted toward the two objectives. The results of the present study can help us better understand the haptic cues involved in object manipulation and shape perception. They also have important implications for the design of future generations of CLDs.

Most haptic systems can be categorized into three groups: vibrotactile displays, force-feedback devices, and fingertip displays. Vibrotactile displays use vibrating elements such as resonant-type tactors to stimulate skin surfaces with lowamplitude, high-frequency vibratory signals. Vibrotactile feedback has been widely used for sensory substitution in the past (e.g., [3]) and more recently, in mobile (e.g., [4], [5]) and wearable (e.g., [6], [7], [8]) applications. Force-feedback 

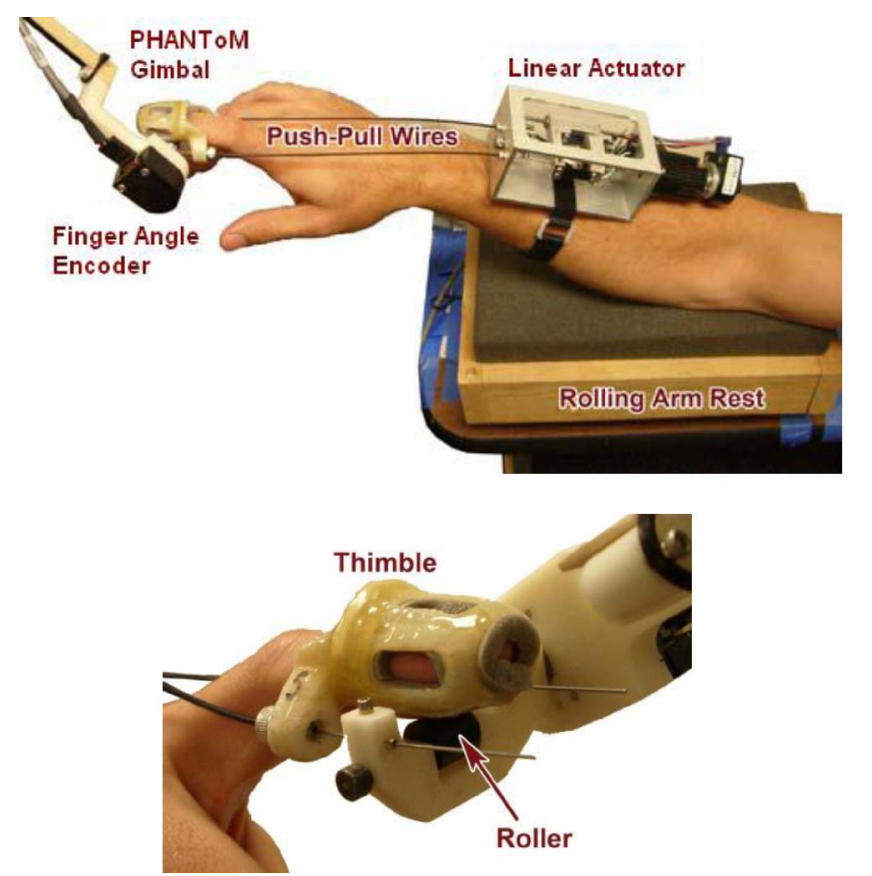

Fig. 1. (Top) Contact location display system. (Bottom) Side view of the roller in the contact location display. ([29, Figs. 3 and 4], respectively.) The user feels the roller on the fingertip through a cut out at the bottom of the thimble. The roller is attached to the PHANToM force-feedback device. The position of the roller relative to the user's fingertip is controlled by the linear actuator worn on the forearm.

devices stimulate the receptors located in muscles, tendons, and joints by imparting forces to the user's hand via a manipulandum (e.g., stylus) or thimble interface. Examples of commercially available force-feedback devices include the PHANToM (Sensable Technology, Woburn, MA), the OMEGA series (Force Dimension, Switzerland) and the Maglev (Butterfly Haptics, Pittsburgh, PA). These devices can simulate the interactions between an interface tool and physical objects in a virtual environment. They are used in a wide range of applications including teleoperation (e.g., [9]), medical training (e.g., [10]), and education (e.g., [11], [12]). A major limitation of both vibrotactile displays and force-feedback devices is that they deprive the user of distributed stress and strain information on the fingertips. Numerous studies have demonstrated the importance of cutaneous information on the fingertips for object identification (e.g., [13]) and tactile shape perception (e.g., [14], [15], [16]). Fingertip displays attempt to restore the missing cutaneous information, and the present study belongs to this growing research area.

The term "fingertip haptics" was first coined by Colgate's group at Northwestern University [17]. Fingertip displays include pin-array devices (e.g., [18]), actuated plates that convey surface orientation and curvature by changing the orientation of a moving plate (e.g., [19], [20]), contact area display (e.g., [21]), slip display (e.g., [17]), contact location display (e.g., [1], [22]), skin stretch displays (e.g., [23], [24], [25]), thermal displays (e.g., [26]), and variable-friction surfaces modulated mechanically (e.g., [27]) or electrically (e.g., [28]). The present study uses the contact location display (see Fig. 1) that was originally developed at Stanford University [1] and has since been slightly improved. The CLD is used to investigate the perception of local features such as corners and small protrusions on object surfaces. It can be mounted on PHANToM force-feedback devices as shown in Fig. 1. As the user moves his/her finger across, say, an edge, the contact point between the fingertip and a roller changes accordingly due to the CLD, thereby augmenting force feedback with tactile contact information. The ability to keep track of the movement of surface features such as edges is expected to facilitate contour following, feature identification, and object manipulation.

The CLD was first used in a study to measure human curvature discrimination thresholds using physical and virtual curvature models. The results showed similar levels of discrimination, indicating the usefulness of the CLD in conveying curvature information [1]. In this initial study, the virtual fingertip was modeled as a line segment and the orientation of the real and virtual fingertips remained horizontal due to a hardware limitation. In a subsequent study [22], the virtual fingertip was modeled as a circular arc, and a rotary encoder was added to the CLD for measuring fingertip orientation. The participants were asked to trace a right-angle corner as quickly as possible without breaking contact. The results indicated that the participants were able to perform the contour following task in less time and with fewer failures (loss of contact) with the CLD. More recent studies have shifted the focus from hardware validation to algorithm development for rendering force and contact location information simultaneously with virtual objects. A new shading algorithm for polygonal object models was developed based on reparameterized Bezier approximations [29]. Participants were asked to discriminate between an ideal smooth cylinder and equivalent polygonal models under various conditions (with and without shading and tactile feedback). The results, reported in terms of the maximum angular changes between adjacent polygons for rendering smooth objects, suggested that the new shading algorithm can significantly reduce a user's sensitivity to discontinuities in polygonal models. Compared to other shading schemes (e.g., [30]), the new shading algorithm can provide both tactile (contact location) and force shading. The thresholds obtained in [29] were incorporated into a 3D force and contact-location rendering platform and evaluated with a 3D object identification task [31].

The present study investigates the effect of CLD on the exploration and perception of shape primitives in touch using two experiments. The next section presents the general methods. The results from the two experiments are reported in Sections 3 and 4, respectively. In the last section, we discuss the implications of our findings.

\section{General Methods}

This section describes the methods that are common to both Experiments 1 and 2. Details specific to each experiment are presented later in Sections 3 and 4, respectively.

\subsection{The Contact Location Display System}

The CLD system displays contact location and contact force simultaneously by combining custom-designed CLD hardware with a PHANToM force-feedback device. A linear 1DOF mechanism is attached to a user's fingertip and forearm to provide contact location information as shown in 


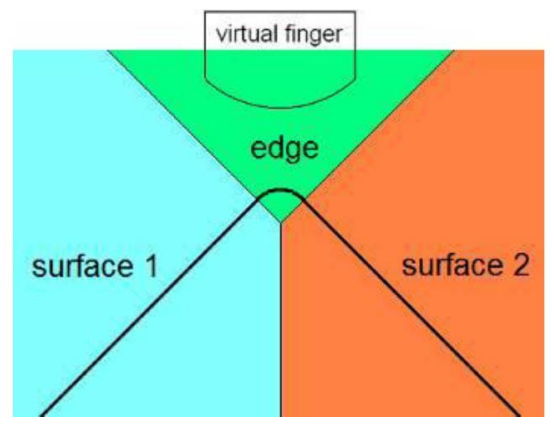

Fig. 2. Illustration of collision calculation regions. ([35, Fig. 2]).

Fig. 1(top). Placement of the linear mechanism on the forearm reduces device inertia at the fingertip and minimizes the transmission of actuator vibrations to the user's fingertip receptors. A user's fingertip is held by an open-bottom thimble, under which a roller is located as illustrated in Fig. 1(bottom). Three different sized interchangeable thimbles were created using rapid-prototyping techniques (fused deposition modeling) to ensure a snug fit for participants with a range of finger sizes. Readings from the position encoders on the PHANToM and CLD are used to calculate the position of the participant's finger. An additional encoder attached to the PHANToM gimbal, shown as the "Finger Angle Encoder" in Fig. 1(top), measures the orientation of the participant's fingertip. The motors of the PHANToM provide reaction forces that are transmitted to the finger through the tactile element (a roller).

The tactile element is a small cylindrical roller with a radius of $4.8 \mathrm{~mm}$ (Experiment 1) or $1.5 \mathrm{~mm}$ (Experiment 2) suspended beneath the participant's fingertip. The thimble and the roller can be moved relative to each other along the distal-proximal direction (i.e., along the length of the fingertip) by two sheathed push-pull wires $(0.61 \mathrm{~mm}$ diameter spring steel). The roller has a fixed range of motion of $16 \mathrm{~mm}$, and a nominal position resolution of $0.17 \mu \mathrm{m}$. A small DC motor actuates the wires via a leadscrew, continuously moving the roller to the appropriate location along the fingertip, as measured by the motor's encoder. While the friction on the push-pull wires varies greatly with the user's hand and finger position, this friction is easily overcome by the torque provided by the leadscrew and DC motor. A pair of the push-pull drive wires positions the contact element along the length of the fingertip and also works as a cantilever spring between the user's finger and the contact element, where the stiffness ranges from 0.16 to $4.3 \mathrm{~N} / \mathrm{mm}$ depending on roller position.

When there is no collision between the user's finger in virtual space and a virtual object, no force is exerted by the PHANToM. The wires prevent the user's fingertip from touching the contact element by working as a cantilever spring. When a collision is detected between the user's finger and an object in virtual space, the roller is moved by the CLD system to the position of contact. The bent wires push the roller against the user's fingertip to render contact. The mechanism can therefore simulate contact and movement of contact location by making and breaking contact between the roller and the user's fingertip. More details about the hardware and the controller can be found in [1] and [22].

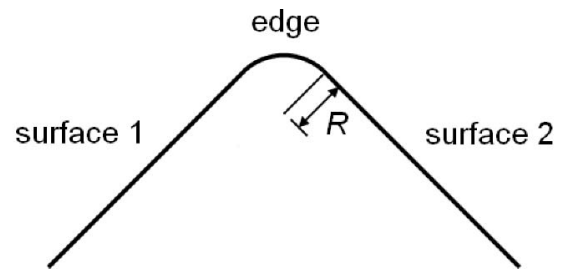

Fig. 3. Illustration of the virtual edges used in the experiment. ([35, Fig. 3]).

\subsection{Haptic Rendering}

The haptic rendering software was developed in Visual C++ using the CHAI3D library (www.chai3d.org). For collision detection, the participant's fingertip was represented in 2D as a circular arc with a radius of $20 \mathrm{~mm}$ (see Fig. 2). The position of the virtual fingertip was updated at $1 \mathrm{kHz}$, based on the participant's fingertip position calculated from the PHANToM's position encoders and the gimbal's finger angle encoder. The entire space was divided into three Voronoi regions [32]. As can be seen in Fig. 2, each region contained one of the three primitives of the virtual object: the edge and the two surfaces. If any part of the virtual finger fell into a region, then the minimum distance between points on the virtual finger and the object surface was calculated. The point on the virtual finger that was closest to the virtual object became a candidate for a possible point of collision. There could be up to three collision-point candidates. The point with the minimum distance to the object surface was selected as the most likely contact point on the fingertip $\left(\mathbf{x}_{\mathbf{f}}\right)$. If this point was on or inside the virtual surface, then a collision was detected, and the virtual finger was replaced by a finger proxy that was constrained to move on the object surface during contact. The concept of a proxy was proposed by Ruspini and Khatib [33], Zilles and Salisbury [34] for calculating penetration depth (and therefore collision force) and to prevent a haptic interface point (HIP) from popping through a virtual object. The collision force was calculated as follows:

$$
\mathbf{F}=K\left(\mathbf{x}_{\mathbf{p}}-\mathbf{x}_{\mathbf{f}}\right),
$$

where $K=3 \mathrm{~N} / \mathrm{mm}$ is the stiffness of the virtual surface; $\mathbf{x}_{\mathrm{p}}$ the proxy position, is the most likely point of collision on the object surface; and $\mathbf{x}_{\mathbf{f}}$ is the most likely contact point on the fingertip (similar to the HIP in haptic rendering algorithms). The target position of the roller was set to $\mathrm{x}_{\mathrm{f}}$. The collision force and the contact location were then displayed through the PHANToM and the CLD devices, respectively.

When no collision was detected, then no force was exerted through the PHANToM device. The target position of the roller, however, was updated in anticipation of an eventual collision. This way, the roller could be positioned at the point of contact on the participant's fingertip without abrupt motion when a collision occurred [22].

\subsection{Stimuli}

The haptic stimuli consisted of a $2 \mathrm{D}$ profile of a radiused edge adjoining two flat surfaces (see Fig. 3). The edge was rendered as a smooth circular arc occupying 90 degree, and the two surfaces as straight lines. The two straight surfaces formed a right angle for all stimuli. The tangent lines at the two ends of the edge had the same slopes as those of the two 


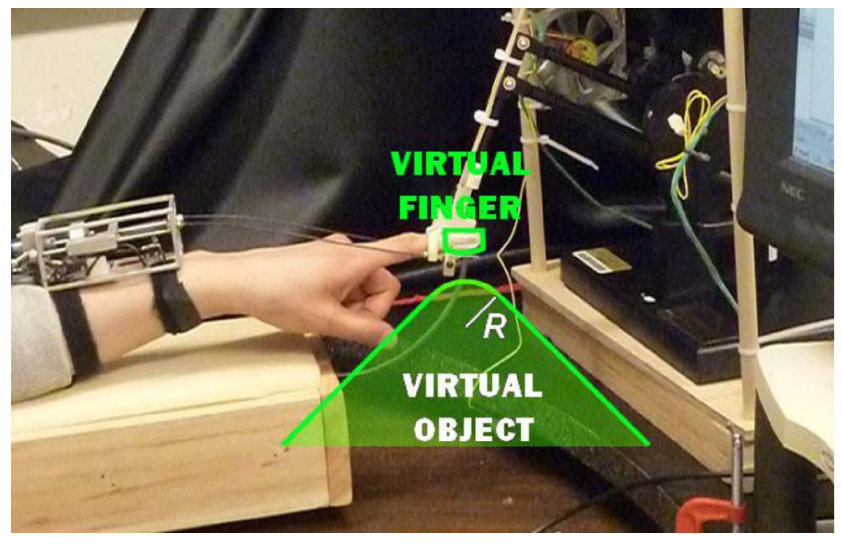

Fig. 4. A photo of the experimental setup. The green overlay illustrates the location of the virtual finger and virtual haptic stimulus. A black cloth covered the PHANTOM, CLD, and participant's hand during the experiments.

surfaces, respectively. The radius of the edge $(R)$ varied from 1.0 to $32.0 \mathrm{~mm}$. The virtual surface was rendered such that the top of the edge was at a constant height regardless of its radius. The haptic stimuli were oriented such that the participants explored the different edges by moving their fingers in fore-aft motions between the two surfaces adjoining the edge (see Fig. 4).

\subsection{Procedures}

The participants discriminated the sharpness of a pair of virtual edges. The method of constant stimuli [36] was used to estimate the discrimination thresholds for edge sharpness. Four different edge radii were selected as the reference stimuli: 2.5, 5.0, 10.0, and $20.0 \mathrm{~mm}$. For each reference stimulus, seven comparison stimuli were selected with equal interstimulus spacing. The spacing for the $2.5 \mathrm{~mm}$ reference radius was $0.5 \mathrm{~mm}$. The spacings for the 5.0, 10.0, and $20.0 \mathrm{~mm}$ reference radii were $1.0,2.0$, and $4.0 \mathrm{~mm}$, respectively. For example, the seven stimulus alternatives for the reference stimulus of $2.5 \mathrm{~mm}$ were 1.0, 1.5, 2.0, 2.5, $3.0,3.5$, and $4.0 \mathrm{~mm}$. On each trial, one of the seven radius values was selected randomly with an equal a priori probability. Both the chosen test stimulus and the constant reference stimulus were presented to the participant on each trial, in a randomly determined order. Each radius value was presented 10 times in a random order, resulting in a total of 70 trials per block of trials at a particular reference radius.

Training was available at the beginning of each block of trials. The participant could see and feel the reference stimulus and all other comparison stimuli by typing a number on the keyboard. The virtual finger was always visible during the training, which was terminated by the participant when she/he was ready. On each trial, the participant explored the reference stimulus and one randomly selected comparison stimulus sequentially. No visual information was shown, except between trials (see below). The participant's task was to indicate which edge was more curved (sharper) by pressing the number key " 1 " (first edge stimulus) or " 2 " (second edge stimulus) as the response. To avoid a large collision force upon the presentation of a new stimulus, the participant was asked to raise his/her finger before entering a response. A horizontal line appeared on

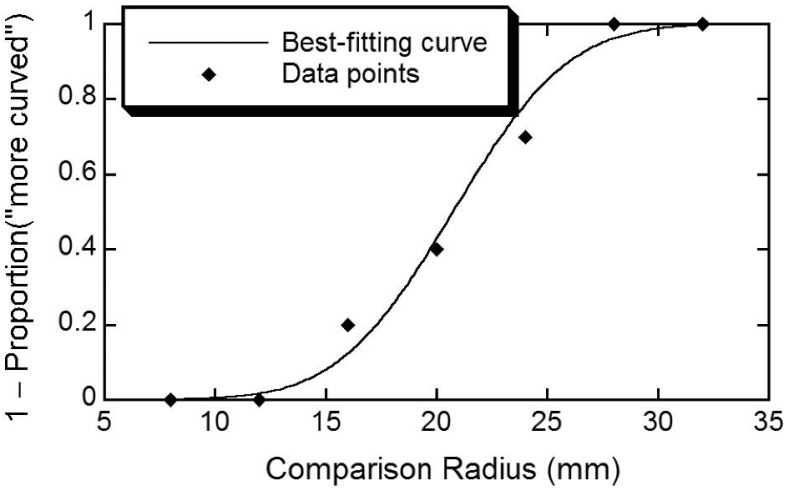

Fig. 5. A representative data plot for one participant at a reference radius of $20.0 \mathrm{~mm}$ from Experiment 1 under the force-only condition. ([35, Fig. 4]).

the screen to indicate how high the finger needed to be lifted, and the virtual finger was shown to indicate the participant's current finger position. After a new stimulus was selected, a green dot was shown on the screen at the top of the curved edge and the participant was asked to lower his/her finger toward the green dot. When the virtual finger came within $2 \mathrm{~mm}$ of the green dot, the horizontal line, the virtual finger, and the green dot disappeared. The participant continued to lower the finger to touch the virtual object. Fig. 4 shows the experimental setup.

The participant wore a pair of earplugs and headphones with a 31-dB noise reduction to block any audio cues from the experimental apparatus. The participant's hand and the experimental apparatus were covered by a black curtain to occlude any possible visual cues. After each block of trials, the participant was instructed to take a 10-min break before continuing. The experiments followed protocols approved by the university IRB.

\subsection{Data Analysis}

For each participant under each condition, the proportion of times that a stimulus was judged to be more curved was tabulated for each reference stimulus. The values of $1-$ P ("more curved") were then fit with an ogive function using the probit analysis tool provided by a SAS software package. The discrimination threshold, or the just noticeable difference (JND), was calculated as the difference between PSE (50 percentile point) and 75 percentile point of the ogive curve resulted from probit analysis. ${ }^{1}$ Fig. 5 shows a representative data plot for one participant.

\section{EXPERIMENT 1: Edge ShARPNESS Discrimination WITH FORCE AND CONTACT LOCATION INFORMATION}

The goal of Experiment 1 was to measure the relative contributions of force and contact location information in edge sharpness perception. We wanted to test the hypothesis that the availability of contact location information would enhance a participant's ability to discriminate the sharpness of virtual edges.

1. Since probit analysis uses a cumulative Gaussian curve to fit the data the same JND value is obtained whether we calculate the difference between the 50 and 75 percentile points, the difference between the 25 and 50 percentile points, or the average of the two differences. 


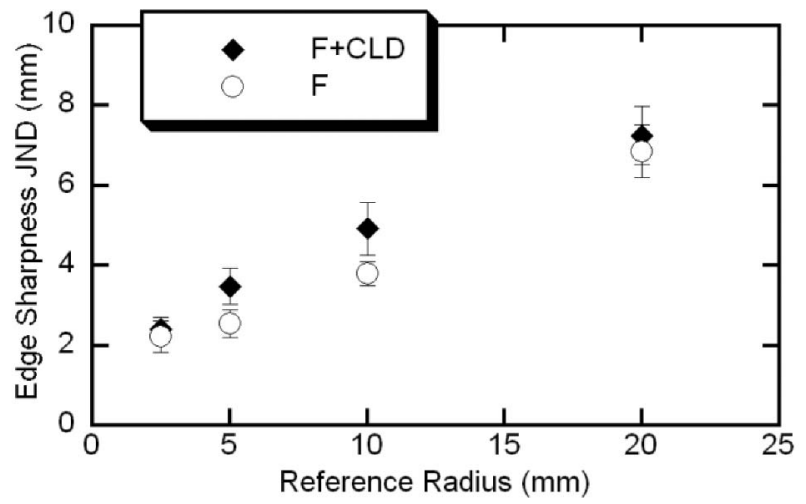

Fig. 6. Means and standard errors of edge sharpness discrimination thresholds for all 14 participants. ([35, Fig. 5]).

\subsection{Participants}

Fourteen participants (eight males and six females, 2345 years old) took part in Experiment 1. Two had prior experience with the CLD and haptic devices. None of them had any known problems with their sense of touch. All were right handed by self-report.

\subsection{Procedures}

Two rendering conditions were used in the experiment: force only $(\mathrm{F})$ and force with contact location display $(\mathrm{F}+\mathrm{CLD})$. In the $\mathrm{F}$ condition, the PHANToM delivered force information calculated from the collision between the virtual finger and the virtual edge and its surrounding surfaces. The roller remained fixed and in contact at the center of the participant's fingertip, thus information about changes in contact location due to the finger's movement on the virtual edge was unavailable. In the F+CLD condition, the roller moved along the participant's fingertip and contacted the fingertip at locations that were consistent with where the virtual edge touched the virtual finger. In the latter case, the participant experienced not only the force delivered by the PHANToM, but also the contact location delivered by the CLD.

Each participant conducted eight blocks of trials (four reference stimuli $\times$ two conditions). The participant took a $10 \mathrm{~min}$ rest between blocks to avoid fatigue. Half the participants completed the F condition first, while the other half completed the F+CLD condition first. The order of the four reference stimuli within each condition was randomized for each participant. It took each participant about 4 hours, conducted over two sessions, to complete Experiment 1.

\subsection{Results}

Eight data plots resembling the one shown in Fig. 5 were obtained for each participant. The JNDs for the same reference stimulus and experimental condition were averaged across the 14 participants. Fig. 6 shows the average data from all 14 participants under both $\mathrm{F}$ and F+CLD conditions as a function of the reference radius. For both conditions, edge sharpness discrimination thresholds increased from 2.3 to $7.4 \mathrm{~mm}$ monotonically when the reference radius increased from 2.5 to $20.0 \mathrm{~mm}$.

A two-way repeated measures ANOVA with the factors experimental condition and reference radius indicates that the reference radius was a significant factor $[\mathrm{F}(3,39)=$ $55.51, p<0.0001]$, but the experimental condition was not
$[\mathrm{F}(1,13)=2.35, p=0.149] .^{2}$ The interaction between the two factors was not significant $[\mathrm{F}(3,39)=0.826, p=0.488]$. A subsequent Tukey test shows that the JNDs for the $F$ condition and F+CLD conditions belonged to the same group (means: 3.9 and $4.5 \mathrm{~mm}$, respectively). Additionally, a pairwise t-test was conducted at each reference radius. None of the threshold pairs was significantly different $[\mathrm{t}(13)=$ $-0.395, \mathrm{p}=0.700 ; \mathrm{t}(13)=-1.890, \mathrm{p}=0.081 ; \mathrm{t}(13)=-1.774$, $\mathrm{p}=0.099 ; \mathrm{t}(13)=-0.517, \mathrm{p}=0.614 ;$ for reference stimuli $2.5,5.0,10.0$, and $20.0 \mathrm{~mm}$, respectively]. During postexperiment debriefing, participants commented that the CLD helped them perceive virtual edges better.

We conclude that edge sharpness discrimination threshold increases with reference radius, and the addition of contact location information does not lead to a significantly different discrimination threshold. This is contrary to our initial expectation that contact location information should enhance edge sharpness perception by lowering the edge sharpness discrimination thresholds. One might still argue that the addition of contact location information should lower edge sharpness discrimination thresholds by noting the JNDs from Provancher et al.'s study [1]. In their study, the experiment condition was similar to the F+CLD condition in the present study and the JNDs were lower than those from the present results. However, it should be noted that Provancher et al.'s study had a constraint in finger motion that might have provided extraneous cues to the participants for edge sharpness discrimination. This was tested and verified by a follow-up experiment presented in the Appendix, which can be found on the Computer Society Digital Library at http://doi.ieeecomputersociety.org/ 10.1109/2012.14. Thus, we still conclude that the addition of contact location information does not enhance edge sharpness perception. We explore the implications of our findings in the next section.

\subsection{A Follow-Up Experiment}

A main objective of Experiment 1 was to investigate the extent to which the CLD benefits the exploration and perception of shape primitives in touch. We anticipated that edge sharpness discrimination thresholds would be lower for the F+CLD condition than for the $\mathrm{F}$ condition. The results, however, indicate that there are no statistically significant differences between the two conditions. There are at least two possible explanations of the results. On the one hand, it is conceivable that both force and contact location cues contribute to the perception of edge sharpness, but force cues dominate the perception. Alternatively, it is possible that contact location information does not benefit edge-shape perception and therefore the addition of CLD does not lead to lower edge sharpness discrimination thresholds. It thus became important to assess the edge sharpness discrimination threshold with contact location information alone, as a way to differentiate the two explanations. To do this, five (three males and two females; one with prior experience with the CLD and haptic devices) of the original 14 participants were randomly selected to take part in a follow-up experiment. Fig. 7 shows a 2D view of the haptic stimuli.

2. An $\alpha$ value of 0.05 was used for all statistical analyses in the present study. 


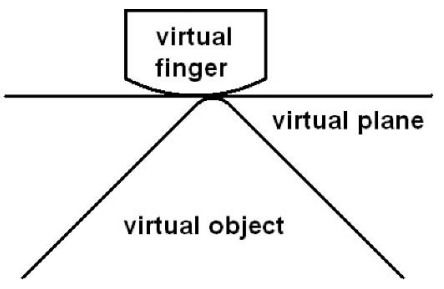

Fig. 7. Illustration of virtual objects used in the CLD alone condition. The virtual plane was used in rendering force. The virtual object was used in calculating contact locations. ([35, Fig. 7]).

Compared to the stimuli used in the main Experiment 1, a flat virtual plane was added to render the resistive force needed in order for the finger to traverse the fore-aft span of the virtual object, yet provide no force information about the shape of the virtual edge that would have occurred if the virtual object's contour was followed. Contact location was rendered through the CLD as described in the main Experiment 1 (i.e., roller position was calculated by projecting finger proxy vertically down on the virtual object contour rather than the virtual plane contour). Note that the edge sharpness discrimination thresholds, so obtained, should be viewed as the upper bounds for the CLD alone condition, as the force cues based on a flat surface could potentially confuse the participants and cause the CLD cues to be less effective in conveying edge curvature information.

The results of the follow-up experiment are plotted in Fig. 8 ("CLD" condition), along with the results from Experiment 1 (data points for " $\mathrm{F}$ " and " $\mathrm{F}+\mathrm{CLD}$ " conditions from Fig. 6). Like the thresholds for the F and F+CLD conditions, the thresholds for the CLD alone condition increased with the reference radius. However, the thresholds for the CLD alone condition were much higher than those for the F or F+CLD condition. A two-way ANOVA with the factors experimental condition and reference radius indicates that both factors were significant [Condition: $\mathrm{F}(2,126)=54.83, p<0.0001$; Radius: $\mathrm{F}(3,126)=25.92, p<$ 0.0001]. A subsequent Tukey test indicates two threshold groups: one for the CLD only condition (mean: $15.6 \mathrm{~mm}$ ) and another for the F and F+CLD conditions (mean: 3.9 and $4.5 \mathrm{~mm}$, respectively). We can conclude that 1) contact location cues do contribute to edge shape perception as indicated by the measurable albeit larger edge sharpness discrimination thresholds for the CLD alone condition; and 2) kinesthetic information dominates edge perception as indicated by the much smaller thresholds for the $\mathrm{F}$ alone condition as compared to the CLD alone condition. Finally, it is observed that the differences between the three test conditions shown in Fig. 8 increased as reference radius increased, indicating an increasing trend of force dominance as the edge became flatter. Conversely, one can argue that the relative contributions of force and contact location cues are more similar for the perception of sharper edges or more localized surface shape primitives.

\section{EXPERIMENT 2: EdGe SHARPNESS Discrimination WITH A 1.5 MM Radius Roller}

The goal of Experiment 2 was to assess the effect of contact roller size on sharpness perception of virtual edges. We hypothesized that a smaller contact roller may lead to a better discrimination performance, due to a greater localization of

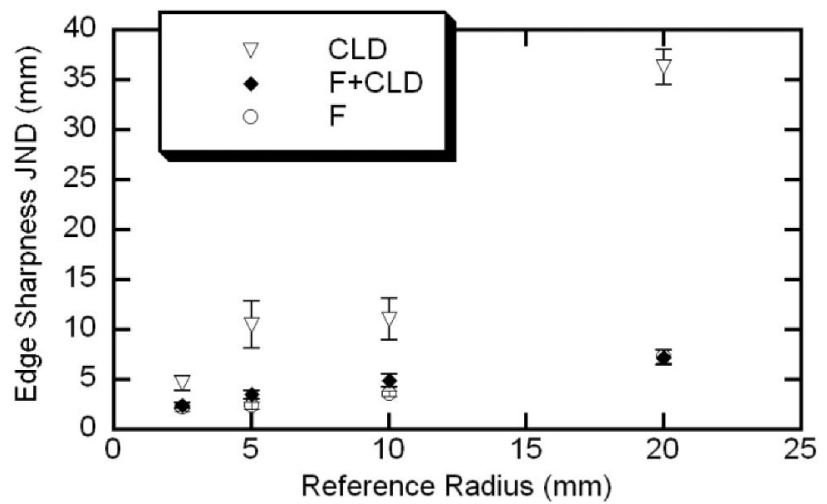

Fig. 8. Comparison of edge sharpness JNDs from the F, F+CLD, and CLD conditions ([35, Fig. 8]). Data for the F and F+CLD conditions are replotted from Fig. 6. Error bars represent standard errors of JNDs.

the moving contact represented by the smaller roller. The contact roller used in Experiment 1 and used previously in the study [1] had a radius of $4.8 \mathrm{~mm}$, which was larger than the radius values for some of the virtual edges rendered. It seemed that the size of the roller conflicted with the contact location movement cues for edges with a radius that was smaller than $4.8 \mathrm{~mm}$. Ideally, the CLD should have a variable-size roller with a radius that instantaneously matches the local curvature of the virtual object. That way, the cutaneous information from physical contact with the roller and the movement of the contact location delivered by the roller would reflect the same virtual surface geometry. Before we redesigned the roller mechanism on the CLD, we asked the question of whether the size of the roller had any effect on edge sharpness discrimination. If the size of the roller was found to significantly affect the perception of edge sharpness, then it would argue for a new CLD with a variable-size roller. In Experiment 2, a new group of participants was recruited to perform the same edge sharpness discrimination task in Experiment 1, except that a smaller roller size was used in the CLD.

\subsection{Participants}

Ten participants (five males and five females, 19 to 32 years old) took part in Experiment 2. None of them had any prior experience with the CLD or haptic devices. None had known problems with their sense of touch. All were right handed by self-report.

\subsection{Procedures}

There was only one condition in Experiment 2. The participants performed an edge sharpness discrimination task with both force and contact location information. The only difference between Experiment 2 and the F+CLD condition of Experiment 1 was the size of the contact roller used in the CLD. The device's roller bracket underwent a minor redesign to accommodate several fixed roller sizes that ranged from 1.5 to $5 \mathrm{~mm}$ in radius, while still keeping the top of each roller in plane the CLD's push-pull wires. The $1.5 \mathrm{~mm}$ radius roller was the smallest size that could easily be produced; hence, it was chosen for this experiment as it was expected to have the greatest effect on perceived edge sharpness.

Each participant conducted four blocks of trials (four reference stimuli) using the method of constant stimuli. The participant took a $10 \mathrm{~min}$ rest between blocks to avoid fatigue. 


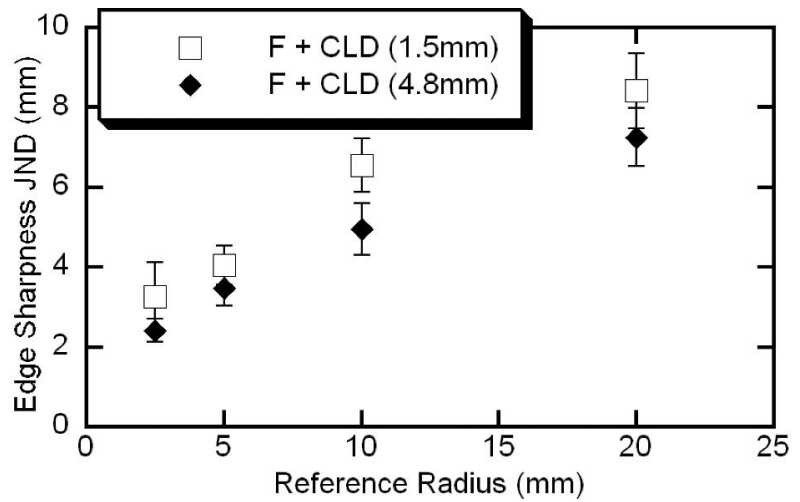

Fig. 9. Comparison of edge sharpness JNDs with a $1.5 \mathrm{~mm}$ (Experiment 2) and a $4.8 \mathrm{~mm}$ (Experiment 1) radius contact roller. The data for the $4.8 \mathrm{~mm}$ roller are replotted from Fig. 6, F+CLD condition. Error bars represent standard errors of JNDs.

The order of the four reference stimuli was randomized for each participant. It took each participant about 2 hours, conducted over one session, to complete Experiment 2.

\subsection{Results}

Four data plots similar to the one shown in Fig. 5 were obtained for each participant. The JNDs for the same reference stimulus and experimental condition were averaged across the 10 participants. Fig. 9 shows the average data from all 10 participants with the $1.5 \mathrm{~mm}$ radius roller along with the data for the $4.8 \mathrm{~mm}$ roller from Experiment 1 for comparison. With the $1.5 \mathrm{~mm}$ radius roller, the edge sharpness discrimination thresholds increased from 3.3 to $8.4 \mathrm{~mm}$ monotonically when the reference radius increased from 2.5 to $20.0 \mathrm{~mm}$. The JNDs with the $1.5 \mathrm{~mm}$ roller tended to be higher than the JNDs obtained in Experiment 1 at all reference radii, but the differences were not statistically significant. A two-way (roller size and reference radius) ANOVA on the data shown in Fig. 9 indicates that reference radius was a significant factor $[\mathrm{F}(3,75)=13.85, p<0.0001]$, but roller size was not $[\mathrm{F}(1,75)=0.66, p=0.4189]$. As in Experiment 1, participants were asked their opinion of the CLD during postexperiment debriefing. All reported that they felt CLD helped them perceive virtual edges better.

The results indicate that the radius of contact roller was not a significant factor for sharpness perception of virtual edges. This is contrary to our expectation that a smaller roller may lead to better discrimination performance due to improved localization of the roller movement.

\section{Discussion and Conclusions}

The present study conducted several edge sharpness discrimination experiments to investigate the cues involved in haptic exploration of small-scale object features and to assess the effect of the size of the CLD contact roller. The results from Experiment 1 showed similar thresholds for the force-only and force-plus-CLD conditions indicating that the addition of contact location information did not significantly improve the participants' ability to discriminate the sharpness of virtual edges. A subsequent experiment with contact location information alone revealed that contact location cues were effective at resolving edge sharpness, although kinesthetic (force+motion) cues dominate edge perception when both cues are present. The discrepancy between the force and contact location cues at conveying edge sharpness information increased as the radius of the edge increased.

Our finding is similar to those from other perception studies where one type of cue can be dominant for the perception of certain object features even though other cues may be available. For example, in haptic texture perception, spatial-intensive (size-depth of microstructures) and vibrational (temporal variations) cues both contribute to texture roughness perception. Although some studies appear to reach seemingly contradictory conclusions [37], [38], [39], [40], [41], [42], an emerging consensus is that humans use vibration cues while exploring surface textures via a probe. While the same temporal cues are available during fingerpad exploration, humans prefer to use spatial and/or intensive cues instead. This is similar to our finding that both force and contact location information contribute to edge sharpness discrimination, but force cues apparently dominate the perception of edge sharpness when both cues are available. More studies are needed to discover when and how contact location information contributes to the perception of local shape primitives.

The results from Experiment 2 showed similar edge sharpness discrimination thresholds for a $1.5 \mathrm{~mm}$ radius roller as compared to the JNDs measured with a $4.8 \mathrm{~mm}$ radius roller in Experiment 1. We conclude that roller size did not have a significant effect on curvature perception in our experiments. This is interesting when we consider the two types of cutaneous cues that are available when a roller moves on a fingertip: the physical contact between the roller and the fingertip provides cutaneous information about the roller's curvature, whereas the movement of the roller on the fingertip provides cutaneous information about the curvature of the virtual surface being rendered. In the present study, these two sources of cutaneous cues were not always consistent except when the stimulus radius was close to 4.8 or $1.5 \mathrm{~mm}$. It appears therefore that the participants were able to overcome (or ignore) the curvature information due to the roller physically contacting the fingertip and instead focus on the roller movement on the fingertip to discern the underlying geometry being conveying with the PHANToM and the CLD. Wijntjes et al. also found that when touched by a flat plate that represented the tangent direction of a virtual curved surface, their participants were able to perceive a curved surface and perform curvature discrimination despite the fact that the plate touching the fingertip was always flat [43].

The results from the present study can be compared to those from previous studies on haptic curvature discrimination. Table 1 lists the Weber fractions from the present study and four other studies that measured haptic curvature discrimination thresholds. We chose to compare Weber fractions instead of JND values because these studies estimated curvature discrimination thresholds at very different reference curvature values, from 0 (flat) to $400 \mathrm{~m}^{-1}$ (2.5 mm in radius). For each study, we plotted the discrimination threshold (JND) as a function of the reference curvature and report the slope of the best fitting line that is passing through the origin as the Weber fraction in Table 1. Despite the fact that these studies used different psychophysical methods and sometimes different performance criteria for defining discrimination thresholds, there is a general 
TABLE 1

Comparison of Weber Fractions for Haptic Curvature Discrimination

\begin{tabular}{ccccc}
\hline $\begin{array}{c}\text { Study } \\
\text { (year) }\end{array}$ & $\begin{array}{c}\text { Real vs. } \\
\text { virtual } \\
\text { curvature }\end{array}$ & $\begin{array}{c}\text { Reference } \\
\text { curvature } \\
\left(\mathbf{m}^{-1}\right)\end{array}$ & $\begin{array}{c}\text { JND } \\
\left(\mathrm{m}^{-1}\right)\end{array}$ & $\begin{array}{c}\text { Weber } \\
\text { fraction }\end{array}$ \\
\hline $\begin{array}{c}\text { Provancher } \\
\text { et al. (2005) [1] }\end{array}$ & Real & $25-100$ & $3.1-7.8$ & 0.08 \\
\hline $\begin{array}{c}\text { van der Horst } \\
\text { et al. (2007) [44] }\end{array}$ & Real & 33 & 2.5 & $\mathbf{0 . 0 8}$ \\
\hline $\begin{array}{c}\text { Goodwin et al. } \\
\text { (1991) [45] }\end{array}$ & $\begin{array}{c}\text { Real } \\
\text { (cutaneous) }\end{array}$ & $0-287$ & $5-32$ & $\mathbf{0 . 1 1}$ \\
\hline $\begin{array}{c}\text { Provancher } \\
\text { et al. (2005) [1] }\end{array}$ & Virtual & $25-100$ & $2.4-11.9$ & $\mathbf{0 . 1 1}$ \\
\hline $\begin{array}{c}\text { Frisoli et al. } \\
\text { (2008) [20] }\end{array}$ & Virtual & 6 & 1.5 & $\mathbf{0 . 2 5}$ \\
\hline $\begin{array}{c}\text { Park et al. } \\
\text { (2011) [35] }\end{array}$ & Virtual & $50-400$ & $13.3-$ & 0.47 \\
\& this study & & & 196.1 & \\
\hline
\end{tabular}

* Weber fraction is the slope of the best fitting line of the JND versus reference function. See text for details.

trend of lower Weber fractions for real curvatures (0.08) than those for virtual curvatures (0.11 to 0.47). As discussed in Section 3.3, the 0.11 Weber fraction obtained by Provancher et al. was likely an underestimate of curvature discrimination threshold due to the limited range of motion that provided additional cues for curvature discrimination (see Appendix, available in the online supplemental material). This further segregates the Weber fraction ranges for real and virtual curvatures. These results imply that the current haptic interfaces need to be further improved in order to deliver more tactile information to the fingertip to improve perception of curved objects.

Efforts are currently underway to develop a 2-DOF version of the CLD for 3D environments in order to provide more tactile information when a participant rotates the finger at a corner or edge. In the future, it will also be interesting to measure curvature discrimination thresholds using real objects with profiles as shown in Fig. 3 to confirm the findings of our study.

\section{ACKNOWLEDGMENTS}

This work was supported in part by the US National Science Foundation Awards under Grant Nos. IIS-0904456 and IIS0904423. Portions of this paper reprinted with permission from [35], (C2011 IEEE.

\section{REFERENCES}

[1] W.R. Provancher, M.R. Cutkosky, K.J. Kuchenbecker, and G. Niemeyer, "Contact Location Displays for Haptic Perception of Curvature and Object Motion," Int'l J. Robotics Research, vol. 24, pp. 691-702, 2005.

[2] B.J. van der Horst and A.M.L. Kappers, "Using Curvature Information in Haptic Shape Perception of 3D Objects," Experimental Brain Research, vol. 190, pp. 361-367, 2008.

[3] J.M. Weisenberger and M.E. Percy, "Use of the Tactaid II and Tactaid VII with Children," The Volta Rev., vol. 96, pp. 41-57, 1994.

[4] J. Pasquero, J. Luk, S. Little, and K. MacLean, "Perceptual Analysis of Haptic Icon: An Investigation into the Validity of Cluster Sorted MDS," Proc. 14th Symp. Haptic Interfaces for Virtual Environment and Teleoperator Systems, pp. 437-444, 2006.
[5] L.M. Brown, S.A. Brewster, and H.C. Purchase, "Multidimensional Tactons for Non-Visual Information Presentation in Mobile Devices," Proc. Eighth Conf. Human-Computer Interaction with Mobile Devices and Services, pp. 231-238, 2006.

[6] H.Z. Tan, R. Gray, J.J. Young, and R. Traylor, "A Haptic Back Display for Attentional and Directional Cueing," Haptics-e: The Electronic J. Haptics Research, vol. 3, 2003.

[7] E. Piateski and L. Jones, "Vibrotactile Pattern Recognition on the Arm and Torso," Proc. World Haptics Conf.: The First Joint Eurohaptics Conf. and Symp. Haptic Interfaces for Virtual Environment and Teleoperator Systems, pp. 90-95, 2005.

[8] J.B.F. van Erp, H.A.H.C. van Veen, and C. Jansen, "Waypoint Navigation with a Vibrotactile Waist Belt," ACM Trans. Applied Perception, vol. 2, pp. 106-117, 2005.

[9] J.J. Abbott and A.M. Okamura, "Pseudo-Admittance Bilateral Telemanipulation with Guidance Virtual Fixtures," Int'l J. Robotics Research, vol. 26, pp. 865-884, 2007.

[10] S. Baillie, A. Crossan, S. Brewster, D. Mellor, and S. Reid, "Validation of a Bovine Rectal Palpation Simulator for Training Veterinary Students," Studies in Health Technology and Informatics, vol. 111, pp. 33-36, 2005.

[11] J. Minogue and G. Jones, "Measuring the Impact of Haptic Feedback Using the SOLO Taxonomy," Int'l J. Science Education, vol. 31, pp. 1359-1378, 2009.

[12] J. Park, K. Kim, H.Z. Tan, R. Refinberger, G. Bertoline, T. Hoberman, and D. Bennett, "An Initial Study of Visuohaptic Simulation of Point-Charge Interactions," Proc. Symp. Haptic Interfaces for Virtual Environments and Teleoperator Systems, pp. 424-430, 2010.

[13] S.J. Lederman and R.L. Klatzky, "Haptic Identification of Common Objects: Effects of Constraining the Manual Exploration Process," Perception and Psychophysics, vol. 66, pp. 618-628, 2004.

[14] A. Frisoli, M. Solazzi, M. Reiner, and M. Bergamasco, "The Contribution of Cutaneous and Kinesthetic Sensory Modalities in Haptic Perception of Orientation," Brain Research Bull., vol. 85, pp. 260-266, 2011.

[15] G. Jansson and L. Monaci, "Identification of Real Objects Under Conditions Similar to Those in Haptic Displays: Providing Spatially Distributed Information at the Contact Areas Is More Important than Increasing the Number of Areas," Virtual Reality, vol. 9, pp. 243-249, 2006.

[16] S. Hsiao, "Central Mechanisms of Tactile Shape Perception," Current Opinion in Neurobiology, vol. 18, pp. 418-424, 2008.

[17] M. Salada, J.E. Colgate, P. Vishton, and E. Frankel, "Two Experiments on the Perception of Slip at the Fingertip," Proc. 12th Int'l Symp. Haptic Interfaces for Virtual Environment and Teleoperator Systems, pp. 146-153, 2004.

[18] I.R. Summers and C.M. Chanter, "A Broadband Tactile Array on the Fingertip," J. Acoustical Soc. of Am., vol. 112, pp. 2118-2126, 2002.

[19] H. Dostmohamed and V. Hayward, "Trajectory of Contact Region on the Fingerpad Gives the Illusion of Haptic Shape," Experimental Brain Research, vol. 164, pp. 387-394, 2005.

[20] A. Frisoli, M. Solazzi, F. Salsedo, and M. Bergamasco, "A Fingertip Haptic Display for Improving Curvature Discrimination," Presence: Teleoperators and Virtual Environments, vol. 17, pp. 550-561, 2008.

[21] E.P. Scilingo, M. Bianchi, G. Grioli, and A. Bicchi, "Rendering Softness: Integration of Kinesthetic and Cutaneous Information in a Haptic Device," IEEE Trans. Haptics, vol. 3, no. 2, pp. 109-118, Apr.-June 2010.

[22] K.J. Kuchenbecker, W.R. Provancher, G. Niemeyer, and M.R. Cutkosky, "Haptic Display of Contact Location," Proc. 12th Int'l Symp. Haptic Interfaces for Virtual Environment and Teleoperator Systems (HAPTICS '04), pp. 40-47, 2004.

[23] K. Bark, J. Wheeler, P. Shull, and J. Savall, "Rotational Skin Stretch Feedback: A Wearable Haptic Display for Motion," IEEE Trans. Haptics, vol. 3, no. 3, pp. 166-176, July-Sept. 2010.

[24] B.T. Gleeson, S.K. Horschel, and W.R. Provancher, "Design of a Fingertip-Mounted Tactile Display with Tangential Skin Displacement Feedback," IEEE Trans. Haptics, vol. 3, no. 4, pp. 297-301, Oct.-Dec. 2010.

[25] J. Luk, J. Pasquero, S. Little, K. MacLean, V. Levesque, and V. Hayward, "A Role for Haptics in Mobile Interaction: Initial Design Using a Handheld Tactile Display Prototype," Proc. ACM Conf. Human Factors in Computing Systems (CHI '06), pp. 171-180, 2006. 
[26] L.A. Jones and H.-N. No, "Warm or Cool, Large or Small? The Challenge of Thermal Displays," IEEE Trans. Haptics, vol. 1, no. 1, pp. 53-70, Jan.-June 2008.

[27] L. Winfield, J. Glassmire, J.E. Colgate, and M. Peshkin, “T-PaD: Tactile Pattern Display through Variable Friction Reduction," Proc. Second Joint EuroHaptics Conf. and Symp. Haptic Interfaces for Virtual Environment and Teleoperator Systems, pp. 421-426, 2007.

[28] O. Bau, I. Poupyrev, A. Israr, and C. Harrison, "TeslaTouch: Electrovibration for Touch Surfaces," Proc. 23rd Ann. ACM Symp. User Interface Software and Technology (UIST '10), pp. 283-292, 2010.

[29] A.J. Doxon, D.E. Johnson, H.Z. Tan, and W.R. Provancher, "Force and Contact Location Shading Thresholds for Smoothly Rendering Polygonal Models," Proc. Symp. Haptic Interfaces for Virtual Environments and Teleoperator Systems, pp. 183-190, 2010.

[30] H.B. Morgenbesser and M.A. Srinivasan, "Force Shading for Haptic Shape Perception," Proc. ASME Dynamics Systems and Control Division, pp. 407-412, 1996.

[31] A.J. Doxon, "Force and Contact Location Shading Methods for Use within Two- and Three-Dimensional Polygonal Environments," master's thesis, Dept. of Electrical and Computer Eng., The Univ. of Utah, Salt Lake City, Utah, 2010.

[32] M. de Berg, O. Cheong, M. van Kreveld, and M. Overmars, Computational Geometry: Algorithms and Applications, third ed. Springer-Verlag, 2008.

[33] D. Ruspini and O. Khatib, "Haptic Display for Human Interaction with Virtual Dynamic Environments," J. Robotic Systems, vol. 18, pp. 769-783, 2001.

[34] C.B. Zilles and J.K. Salisbury, "A Constraint-Based God-Object Method for Haptic Display," Proc. Int'l Conf. Intelligent Robots and Systems, pp. 146-151, 1995.

[35] J. Park, A.J. Doxon, W.R. Provancher, D.E. Johnson, and H.Z. Tan, "Edge Sharpness Perception with Force and Contact Location Information," Proc. World Haptics Conf., pp. 517-522, 2011.

[36] G.A. Gescheider, Psychophysics: The Fundamentals, third ed. Lawrence Erlbaum Assoc., 1997.

[37] D. Katz, The World of Touch. Lawrence Erbaum Assoc., 1925/1989.

[38] M.M. Taylor and S.J. Lederman, "Tactile Roughness of Grooved Surfaces: A Model and the Effect of Friction," Perception and Psychophysics, vol. 17, pp. 23-36, 1975.

[39] C.E. Connor and K.O. Johnson, "Neural Coding of Tactile Texture: Comparison of Spatial and Temporal Mechanisms for Roughness Perception," J. Neuroscience, vol. 12, pp. 3414-3426, 1992.

[40] S.J. Lederman, R.L. Klatzky, C.L. Hamilton, and G.I. Ramsay, "Perceiving Roughness via a Rigid Probe: Psychophysical Effects of Exploration Speed and Mode of Touch," Haptics-e: The Electronic J. Haptics Research, vol. 1, pp. 1-20, 1999.

[41] K.O. Johnson and S.S. Hsiao, "Neural Mechanisms of Tactual Form and Texture Perception," Ann. Rev. Neuroscience, vol. 15, pp. 227-250, 1992.

[42] S.J. Lederman and R.L. Klatzky, "Sensing and Displaying Spatially Distributed Fingertip Forces in Haptic Interfaces for Teleoperator and Virtual Environment Systems," Presence: Teleoperators and Virtual Environments, vol. 8, pp. 86-103, 1999.

[43] M.W.A. Wijntjes, A. Sato, V. Hayward, and A.M.L. Kappers, "Local Surface Orientation Dominates Haptic Curvature Discrimination," IEEE Trans. Haptics, vol. 2, no. 2, pp. 94-102, Apr.-June 2009.

[44] B.J. van der Horst and A.M.L. Kappers, "Curvature Discrimination in Various Finger Conditions," Experimental Brain Research, vol. 17, pp. 304-311, 2007.

[45] A.W. Goodwin, K.T. John, and A.H. Marceglia, "Tactile Discrimination of Curvature by Humans Using Only Cutaneous Information from the Fingers," Experimental Brain Research, vol. 86, pp. 663-672, 1991.

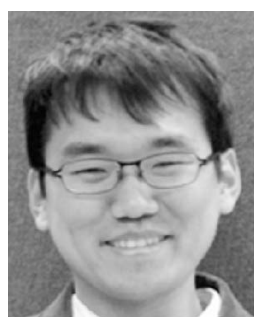

Jaeyoung Park received the BS degree in electrical and computer engineering from Seoul National University, Korea, and the MS degree in electrical and computer engineering from Purdue University, West Lafayette, Indiana. He is currently working toward the $\mathrm{PhD}$ degree in electrical and computer engineering at Purdue University. $\mathrm{He}$ is a student member of the IEEE.

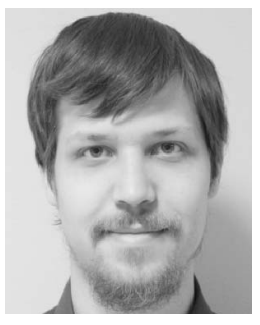

Andrew J. Doxon received the BS degree in electrical engineering from New Mexico Technological University in 2008 and the MS degree in electrical engineering from the University of Utah in 2010. His master's thesis focused on developing new haptic shading algorithms to allow tactile feedback devices to operate smoothly in polygonal environments. $\mathrm{He}$ is currently continuing his masters' research while pursuing the $\mathrm{PhD}$ degree in mechanical engineering from the University of Utah. He is a student member of the IEEE.

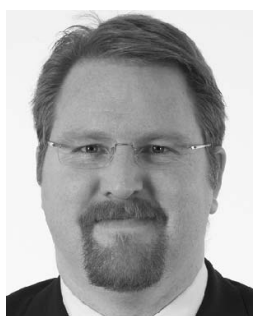

William R. Provancher received the BS degree in mechanical engineering and the MS degree in materials science and engineering, both from the University of Michigan. He received the PhD degree from the Department of Mechanical Engineering, Stanford University, in the area of haptics, tactile sensing, and feedback. His postdoctoral research involved investigating and designing bioinspired climbing robots, focusing on creating robot foot designs for climbing vertical surfaces with compliantly supported microspines. He is currently a tenured associate professor in the Department of Mechanical Engineering, University of Utah. He teaches courses in the areas of mechanical design, mechatronics, and haptics. His active areas of research include haptics, tactile feedback, and the design of novel climbing robots. He received the US National Science Foundation (NSF) CAREER award in 2008 and has won the Best Paper and Poster Awards at the 2009 and 2011 World Haptics Conferences for his work on tactile feedback. He is a member of the IEEE. Research details and related publications are linked on his homepage: http://www.mech. utah.edu/people/faculty/provancher.html.

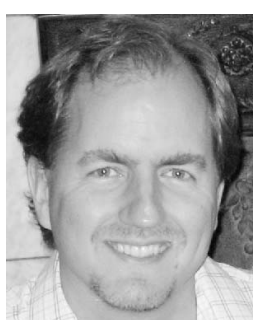

David E. Johnson received the BA degree in computer science and physics from Carleton College and the $\mathrm{PhD}$ degree in computer science from the University of Utah, focusing on geometric computations for haptic rendering. $\mathrm{He}$ is a research scientist at the University of Utah's School of Computing. His current interests are in applying geometric computations to the area of robotics.

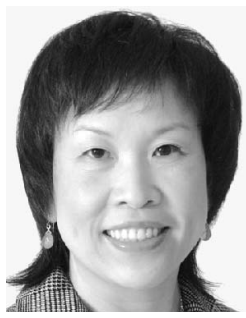

Hong Z. Tan received the bachelor's degree in biomedical engineering in 1986 from Shanghai Jiao Tong University and the master and doctorate degrees in 1988 and 1996, respectively, both in electrical engineering and computer science, from the Massachusetts Institute of Technology (MIT). She was a research scientist at the MIT Media Lab from 1996 to 1998 before joining the faculty at Purdue University. She is currently an associate professor of electrical and computer engineering, with courtesy appointments in the School of Mechanical Engineering and the Department of Psychological Sciences. She is an associate editor of Presence, the ACM Transactions on Applied Perception, and the IEEE Transactions on Haptics. Shee served as the founding chair of the IEEE Technical Committee on Haptics from 2006 to 2008 . She was the recipient of the US National Science Foundation (NSF) CAREER award from 2000 to 2004. Her research focuses on haptic human-machine interfaces in the areas of haptic perception, rendering and multimodal performance. She is a senior member of the IEEE.

$\triangleright$ For more information on this or any other computing topic, please visit our Digital Library at www.computer.org/publications/dlib. 